\title{
Trastorno específico del lenguaje (TEL): concepto y características.
}

(Specific language impairment (SLI): concept and features.)

\author{
Laura González Blanca \\ Universidad de Jaén, España.
}

Fecha recepción: 10-08-2018

Páginas 166-174

Fecha aceptación: 30-09-2018

\section{Resumen.}

En este artículo, y dada la importancia que está tomando este trastorno en la actualidad, me veo en el compromiso de realizar una revisión bibliográfica sobre el trastorno específico del lenguaje, TEL de aquí en adelante. Este trastorno ha sido explicado por diversos autores que le pretenden dar con sus aportaciones un significado y dotarlo de unas características claras. Para ello, veremos su conceptualización, una breve clasificación, el tratamiento educativo que debería darse hacia el TEL y su intervención. Por último, a modo de conclusión, aporto un estudio realizado por Van der Lely y Howard en 1993. Este estudio trata de aclarar que es lo que les ocurre los niños/as que padecen TEL, si es un deterioro en el lenguaje 0 un déficit en el funcionamiento de la memoria a largo plazo.

Palabras clave: trastorno; específico; lenguaje; intervención; TEL

\begin{abstract}
.
In this article, and given the importance that this disorder is taking nowadays, I am in the commitment to do a bibliographic review about the specific language impairment, SLI from now on. This disorder has been explained for various authors who intend to give some meaning with their contributions and provide it with clear characteristics. For that, we will see the concepts, a short classification, the educational treatment and the educational intervention that has been done. As a conclusion, I contribute a study of Van der Lely and Howard in 1993. This study aims to clear out what happened to the children who have SLI, if it is a deterioration in the language or a deficit in the long-term memory operation.
\end{abstract}

Keywords: disorder; specific; language, intervention; SLI 


\section{1.-Introducción.}

En una situación normal, podemos contemplar la idea de que un niño aprende el lenguaje mediante su entorno psicosocial y las relaciones que mantenga con la familia, primer contacto con el que se relaciona, de esta forma, también se le permitirá acceder al lenguaje interno para acceder al aprendizaje. Pero el gran interrogante es la forma en la que un niño descubre las reglas gramaticales, el léxico, los sonidos y la interrelación entre ellos para adquirir el lenguaje que es lo que permitirá la comunicación. Así pues, la American Speech-Language-Hearing (ASHA, 2010) define el lenguaje como un sistema complejo y dinámico de signos interrelacionados que permiten la comunicación, parece ser una habilidad innata.

En torno al lenguaje, existen varios conceptos importantes los cuales debemos tener en cuenta:

- Fonología: sonidos que permiten formar palabras. La unidad mínima capaz de producir las diferencias en el significado es el fonema.

- Morfología: se refiere a la forma que toman las palabras. La sintaxis nos indica la forma en la que se pueden combinar las palabras para formar diferentes oraciones.

- Semántica: es el significado que tienen las palabras y frases que reproducimos.

- Pragmática: es el uso afectivo que le damos al lenguaje.

- Léxico: es el vocabulario que maneja cada sujeto. Puede ser diferente en cada persona. Hablamos de jergas para referirnos al vocabulario específico de un grupo de personas o de profesionales; este vocabulario es usado cuando los hablantes se reúnen.

\section{1.-Dimensión social del lenguaje.}

El lenguaje es el primer y principal objeto a la hora de llevar a cabo la comunicación y el desempeño académico; es por lo tanto fundamental para La socialización. Las habilidades sociales y las destrezas del lenguaje trabajan en una interacción conjunta para que la competencia social se desarrolle y, por lo tanto, se creen unos vínculos en las relaciones tanto con pares como con profesores.

Otra parte fundamental del lenguaje es que lo niños se expresan, formulan deseos, creencias y necesidades a través de la comunicación, por lo que será esencial un buen entendimiento y práctica del mismo para conseguir lo deseado.

\section{2.-Desarrollo del lenguaje.}

La adquisición del lenguaje es uno de los logros mayores que presentan los niños desde pequeños y aunque muchas de esas veces existen casos en los que no hay mayor dificultad (la mayoría), hay otros en los que se presentan dificultades. Estas dificultades varían respecto a la persona en la que nos estemos centrando, por lo que su etiología, denominación clínica, características y pronóstico será muy variable. 


\section{2.-Conceptualización.}

El concepto de este trastorno nace como evolución de otros los cuales podríamos considerar relacionados con el mismo o como una derivación de los trastornos afásicos. Estos se producen por consecuencia de una patología cerebral. Las afasias son pérdida de la capacidad de producir o comprender el lenguaje, debido a lesiones en áreas que se especifican en áreas del lenguaje que se especializan en estas funciones.

La evolución del concepto parte con Benton (1964) que consideró "la afasia infantil como un trastorno evolutivo caracterizado por presentar problemas severos de comprensión y/o expresión del lenguaje hablado, en ausencia de pérdida auditiva, retraso mental o trastorno emocional.

Años más tarde, Launay (1975) se refirió a la disfasia para referirse a "un trastorno funcional, sin substratum de lesión orgánica clínicamente descubrible [...] La disfasia funcional es, para nosotros, una forma de evolución del déficit de lenguaje observado en los primeros años de la vida, todos los estadios intermedios existentes entre un retraso totalmente curable y las formas más duraderas de la disfasia". Lunay usó diferentes términos para referirse a trastornos más graves de adquisición y elaboración del lenguaje, los cuales son afasia congénita de expresión, audiomudez, afasia de recepción, sordera verbal y agnosia auditiva.

La definición hasta el momento que encontramos más característica sobre el TEL es de la American Speech-Language-Hearing Association, ASHA (1980): "un trastorno de lenguaje es la anormal adquisición, comprensión o expresión del lenguaje hablado 0 escrito. El problema puede implicar a todos, uno o algunos de los componentes fonológico, morfológico, semántico, sintáctico o pragmático del sistema lingüístico. Los individuos con trastornos del lenguaje tienen frecuentemente problemas de procesamiento del lenguaje 0 abstracción de la informacion significativa para almacenamiento y recuperación por la memoria a corto o a largo plazo".

Tras estos, el avance más significativo que encontramos en la delimitación de la conceptualización del TEL se debe a los estudios de Stark y Tallal (1981). Los autores marcaron unos criterios para la clasificación de los niños con TEL y la exclusión de aquellos cuyos problemas en el lenguaje de se deben a una causa diferente. Según Stark y Tallal (1981) estos criterios son:

- Nivel auditivo de 25db en la banda de frecuencias de 250 a $6.000 \mathrm{~Hz}$, y de 25 en el reconocimiento de palabras familiares.

- Estatus emocional y conducta normal, por lo que se excluyen los casos que se presenten problemas conductuales severos o problemas especiales de ajuste familiar o escolar.

- Nivel intelectual mínimo, por lo que se considera que no presentan este trastorno los niños cuyo $\mathrm{Cl}$ de ejecución sea inferior a 85. 
- Estatus neurológico sin signos de alteración, por lo que no alcanzarían el criterio de TEL los niños con claros signos neurológicos o con historia de trauma cerebral, epilepsia u otros marcadores de trastorno neurológico.

- Destrezas motoras del habla normales, con exclusión de los niños con problemas orales motores periféricos, deficiencia en la sensibilidad oral 0 anormalidades orofaciales.

- Nivel lector normal, en caso de que el niño haya iniciado el aprendizaje formal de la lectura.

\section{3.-Clasificación.}

La clasificación posiblemente más demandada y una de las más aceptadas es la realizada por Rapin y Allen (1987):

Tabla 1: Clasificación de los tipos de trastornos del lenguaje.

\begin{tabular}{|l|l|}
\hline $\begin{array}{l}\text { A.- Trastornos específicos del } \\
\text { lenguaje }\end{array}$ & $\begin{array}{l}\text {-Dispraxia verbal } \\
\text {-Déficit de programación fonológica }\end{array}$ \\
\hline $\begin{array}{l}\text { B.- Trastornos del lenguaje expresivo } \\
\text { y receptivo }\end{array}$ & -Agnosia auditivo-verbal \\
\hline $\begin{array}{l}\text { C.- Trastornos del procesamiento de de } \\
\text { orden superior }\end{array}$ & -Déficit fonológico sintáctico \\
\hline
\end{tabular}

Fuente: Rapin y Allen (1987)

La taxonomía de la Organización Mundial de la Salud (OMS) recopila de manera diferenciada los trastornos específicos del desarrollo del lenguaje excluyendo los trastornos adquiridos y/o con causa neurológica conocida.

Tabla 2: Trastornos específicos del desarrollo del habla y del lenguaje

Trastornos específicos del desarrollo del habla y del lenguaje

1. Trastorno específico del lenguaje

2. Trastorno de la expresión del lenguaje

3. Trastorno de la comprensión del lenguaje

4. Afasia adquirida por epilepsia

5. Otros trastornos del desarrollo del habla y del lenguaje

6. Trastornos del desarrollo del habla y del lenguaje sin especificación

Fuente: Organización Mundial de la Salud (OMS)

\section{4.-Tratamiento educativo e intervención.}

El retraso en el desarrollo del lenguaje con respecto a otras destrezas es una las dificultades más frecuentes en los años preescolares. Distintas observaciones han mostrado que los niños que presentan retraso en las primeras adquisiciones lingüísticas constituyen un grupo de riesgo de problemas posteriores de lenguaje y aprendizaje. Si se postula la existencia de un período crítico (Lenneberg, 1967), los tres primeros años de vida son de vital importancia para moldear el futuro desarrollo 
social e intelectual, y la intervención temprana sobre las dificultades iniciales tendrá un carácter preventivo posterior.

Después de identificar el retraso en el desarrollo de los niños de cada una de las tres dimensiones evaluadas, Ward (1999) diseñó un programa para intervenir sobre estos retrasos encontrados y consistían en proporcionar a los niños una estimulación ambiental suficiente y de una calidad que pudieran beneficiarse de ella. Era necesario aprovechar las situaciones naturales como input de las formas lingüísticas de interés. Los programas desarrollados son los siguientes:

\section{Grupo 1. Retraso expresivo y receptivo asociado a dificultades auditivas generalizadas.}

Esta hipótesis se basaba en que el fracaso para desarrollar la habilidad de atender de forma selectiva a los sonidos se debía a las dificultades auditivas y al ruido de fondo (ruido ambiental normal). Se considera esta habilidad de máxima importancia para desarrollar la competencia lingüística y que debe estar adquirida al final del primer año de vida. Las variables que afectan al desarrollo son las siguientes:

- Excesivo ruido.

-Pérdidas conductivas frecuentes: infecciones frecuentes de las vías respiratorias.

- Estilo de habla adulto hacia el niño.

- Grado de estimulación ambiental.

\section{Grupo 2. Retraso expresivo y receptivo.}

Los niños desarrollan unas destrezas auditivas adecuadas aunque presentan problemas para comprender el mensaje verbal. Ward formuló que se cambiaba la cantidad y calidad verbal de los cuidadores facilitarían la comprensión verbal en los niños. Los objetivos que plantea son los siguientes:

-Promover el interés del niño por escuchar la voz, proporcionándoles una estimulación adecuada.

-Desarrollar la comprensión verbal.

-Incrementar la cantidad de interacción y darles a los niños oportunidades para percibir los aspectos prosódicos del habla.

\section{Grupo 3. Retraso expresivo.}

La cantidad de información que reciben los niños y niñas no tiene la calidad suficiente para permitirles desarrollar la habilidad de discriminar sonidos correctamente. Como objetivos concretos Ward propone los siguientes:

- Generar una adecuada atención compartida entre los niños y los cuidadores. -Imitar los sonidos ambientales para aumentar el interés por los sonidos. 
-Repetir varias veces los mensajes y las palabras para que los niños y niñas se familiaricen con ellas y así poder recordarlas.

Algunas sugerencias que podríamos seguir a la hora de intervenir sobre estos problemas podrían ser los siguientes:

-Si nos encontramos con una situación en la que hay ruido excesivo podríamos programar ratos de silencio, hacer juegos sonoros o mostrarle a los niños y niñas las fuentes sonoras.

-Por otro lado, si nos encontramos con pérdidas frecuentes auditivas podríamos tratar esta situación mediante una serie de técnicas como pueden ser hablar siempre cerca del niño o niña o hablarle un poco más fuerte.

-En cambio, si la estimulación ambiental es escasa o padecen defensa perceptiva es aconsejable no reñir verbalmente, no pedirle al niño o niña que emita palabras y, si lo hace, no corregirle si las dice mal.

Hay que tener en cuenta el estilo de habla entre cuidador o cuidadora y niño o niña puesto que es necesario hablar de una forma concreta usando frases cortas, hablar despacio, enfatizar la entonación o utilizar el gesto, entre otros.

\section{5.-Conclusión.}

A modo de conclusión, he elegido el estudio de Van der Lely y Howard (1993), que servirá para evidenciar empíricamente El estudio sobre el trabajo de la memoria en niños con TEL.

Este estudio pretende aclarar si los niños diagnosticados con TEL tienen un deterioro del lenguaje 0 un déficit en la memoria a corto plazo (Van der Lely y Howard, 1993).

La literatura previa nos muestra estudios como el de Kirchner y Klatzly (1985), los cuales investigaron con ensayos verbales en niños TEL y compararon esta ejecución con un grupo de niños igualados en edad cronológica. Se le presentan los ítems y los niños ensayan cada ítem en voz alta. Desde la fase de ensayo hasta la de ejecución del recuerdo se evaluaron 12 variables tales como retención y orden de los ítems, organización semántica, repetición e intrusión de errores.

La diferencia predominante entre niños TEL y el grupo de los otros niños fue en capacidad de retención y regeneración de ítems e intrusión de errores, lo que los autores interpretaron como que los TEL difieren respecto a los controles en su capacidad en memoria a corto plazo. Haciendo un análisis más detallado se puede decir que estos hallazgos se producen por una disminución de la capacidad verbal en la memoria a corto plazo.

Dichos autores continuaron con otro estudio basado en la rapidez de la memoria en registrar, para poder especificar más el foco del déficit. Para ello el niño tiene que identificar si un ítem aparece en una lista de dígitos que ha sido previamente 
presentada de forma verbal. La rapidez en decir sí o no proporciona la medida de rapidez del registro. Este estudio demostró que los niños TEL son cuatro veces más lentos en el registro en la memoria a corto plazo que sus iguales con desarrollo lingüístico normal.

Van der Lely y Haward (1993) estudiaron el procesamiento y la representación lingüística en relación con la MCP de los niños TEL comparándolo con niños igualados en edad y cuyo estadio lingüístico era parecido. Es bien conocido que los niños en distintos niveles de desarrollo usen estrategias diferentes para completar tareas experimentales, estas diferencias en ejecución, tanto en tareas de lenguaje como en tareas de MCP deben reflejar el uso de diferentes procesos del lenguaje subyacentes, en los distintos estadios del desarrollo. Esta comparación, argumentan los autores, que es mejor porque revela áreas de déficit específicas y selectas que están desproporcionadamente deterioradas en relación con sus niveles de desarrollo lingüístico general. En cambio una comparación llevada a cabo solo con niños controles de la misma edad con desarrollo normal revela poco acerca de la naturaleza de los deterioros lingüísticos de la población TEL ya que los niños TEL ejecutarán siempre por debajo las tareas en las que el desarrollo lingüístico se vea envuelto.

El deterioro de lenguaje de los niños TEL debe interactuar con diferentes componentes del lenguaje y además puede afectar hasta cierto punto a muchas, si no a todas las funciones del lenguaje. Se pretende responder a las siguientes cuestiones en el estudio de Van der Lely y Howard (1993):

- ¿La ejecución de tareas en la memoria a corto plazo de los niños con TEL difiere de la de sus iguales en el nivel lingüístico?

-Si es así, ¿el patrón de ejecución a través de varias tareas difiere para los niños con TEL en comparación con sus iguales?

Este estudio compara la MCP de niños TEL (identificados por el criterio de inclusión) y controles igualados en edad y en una serie de medidas de lenguaje (LA). Las tareas utilizadas fueron la repetición de listas de palabras y de señalización de un dibujo. En este estudio las características lingüísticas de los estímulos de los test fueron designadas para explorar la influencia de la similitud semántica, léxica y fonológica en la MCP. El paradigma de repetición requiere salida verbal, almacenamiento y procesamiento. La respuesta de señalar requiere solo almacenamiento y procesamiento pero no salida verbal. Se hipotetiza que si alguno de estos tres procesamientos (semántico, léxico o fonológico) estuviera involucrado en el recuerdo inmediato, entonces el recuerdo de palabras similares (semánticamente, lexicalmente o fonológicamente) debería ser más pobre que el recuerdo de palabras no relacionadas. Por tanto si los niños TEL estuvieran utilizando alguno de estos dos procesamientos en mayor medida que los niños normales, entonces se apreciarían diferencias entre los dos grupos. El estudio se divide en tres experimentos: 


\section{1.-Procesamiento cognitivo semántico.}

Se comparó el recuerdo para palabras similares y no similares semánticamente. Baddeley encontró que el recuerdo fue peor para las palabras semánticamente similares que para las palabras no relacionadas tanto para el recuerdo inmediato como para el demorado. Si los TEL están usando procesamiento semántico en la MCP para el recuerdo de palabras, debe de haber diferencias entre los dos grupos en la ejecución de las tareas de recuerdo. Había dos tareas en una se pedía que se repitiera las palabras de una lista (paradigma de repetición) y en la otras señalar las palabras en un dibujo, que el examinador previamente decía. Se tuvo también en cuenta si el recuerdo era ordenado o no en ambas tareas.

Los resultados del experimento indican que los niños TEL parecen estar ejecutando en un nivel similar y en la misma dirección que lo hicieron los normales igualados en comprensión y expresión del lenguaje. Esto sugiere que los TEL no dependen más del procesamiento semántico de las palabras que sus iguales controles.

\section{2.-Efecto del procesamiento léxico.}

Se exploran los efectos del procesamiento léxico y representación en el recuerdo serial inmediato en niños TEL y controles. La ejecución en sets de palabras y pseudopalabras se comparó. Se predijo que si el conocimiento léxico se usara para facilitar el recuerdo, la ejecución con pseudopalabras debería ser más pobre que con palabras reales. En ambos grupos las palabras se recordaron mejor que las pseudopalabras esto quiere decir que los dos grupos fueron sensibles a las características del material. Este experimento demuestra que para el recuerdo inmediato de palabras no relacionadas y pseudopalabras, los niños TEL ejecutan de forma similar a los controles.

\section{3.-Procesamiento fonológico.}

El procesamiento fonológico en MCP en niños TEL se investigo comparando la ejecución en recuerdo inmediato de palabras no relacionadas y similares fonológicamente usando tanto el paradigma de repetición como el de señalar dibujos. Se comprobó que las palabras y pseudopalabras relacionadas fonológicamente se recordaron peor que las que no lo estaban. Este efecto ha sido atribuido a la separación de los componentes de la MCP: el lazo articulatorio y el almacén fonológico. Este experimento va dirigido a investigar la habilidad de almacenamiento de la memoria a corto plazo fonológica de los TEL pero no del lazo articulatorio.

El efecto de similitud fonológica fue significativo para ambos paradigmas. Se refleja la baja ejecución para las palabras fonológicamente similares. Los dos grupos parecen similares en sus recuerdos de materiales en los dos paradigmas. Los niños TEL y los controles parecen estar afectados de la misma forma por las características fonológicas de los materiales indicando, que los niños TEL tienen una capacidad 
para el material fonológico y un almacenamiento similar para dicho material que los niños controles.

\section{6.-Referencias.}

American Psychiatric Association (2000) . Diagnostic and statistical manual of mental disorders, text revised (DSM-IV TR). Washington DC: APA; 2000.

Benton, A. L. (1964). The memoir of Marc Dax oc Aphasia. Neurology, 14, 851-4.

Kirchner, D. \& Klatzly, R. (1985). Verbal rehearsal and memory in language disordered children. Journal of Speech and Hearing Disorders, 28,556-565.

Lennenberg, E. H. (1967). Biological Foundations of Language. NY: John Wiley and Sons Inc.

Rapin, I., Allen, D. A. (1987). Developmental language disorders: nosolgic considerations. New York: Academic Press.

Stark, R. E., Tallal, P. (1981). Selection of children with specific language deficits. Journal of Speech and Hearing Disorders, 46, 114-122.

Van der Lely, H. K. J., Howard, D. (1993). Children with specific language impairment: Linguistic impairment or short-term memory deficit? Journal of Speech \& Hearing Research, 36(6), 1193-1207.

Ward, S. (1999). An investigation into the effectivennes of an early intervention method for delayed language development in young children. International Journal for Language and Communication Disorder. 34, 243-246. 